\title{
ICIPEMIR: Improving the Completeness, Interoperability and Patient Explanations of Medical Imaging Reports
}

\author{
Arthur LAURIOT DIT PREVOST ${ }^{\mathrm{a}, \mathrm{b}, 1}$ Marie TRENCART ${ }^{\mathrm{a}, \mathrm{b}}$, Vianney GAILLARD $^{\mathrm{c}}$, \\ Guillaume BOUZILLE ${ }^{\mathrm{d}}$, Rémi BESSON ${ }^{\mathrm{a}, \mathrm{b}}$, Dyuti SHARMA ${ }^{\mathrm{a}, \mathrm{b}}$, Philippe PUECH ${ }^{\mathrm{c}}$, \\ and Emmanuel CHAZARD ${ }^{\mathrm{a}}$ \\ ${ }^{a}$ Univ. Lille, CHU Lille, ULR 2694 METRICS, F-59000 Lille, France \\ ${ }^{\mathrm{b}} \mathrm{CHU}$ Lille, Clinique de Chirurgie et Orthopédie de l'Enfant, F-59000 Lille, France \\ ${ }^{\mathrm{c}}$ Univ. Lille, Inserm, CHU Lille, U1189 - ONCO-THAI, F-59000 Lille, France \\ ${ }^{\mathrm{d}}$ Univ Rennes, CHU Rennes, Inserm, LTSI - UMR 1099, F-35000 Rennes, France
}

\begin{abstract}
Introduction. Although electronic health records have been facilitating the management of medical information, there is still room for improvement in daily production of medical report. Possible areas for improvement would be: to improve reports quality (by increasing exhaustivity), to improve patients' understanding (by mean of a graphical display), to save physicians' time (by helping reports writing), and to improve sharing and storage (by enhancing interoperability). We set up the ICIPEMIR project (Improving the completeness, interoperability and patients explanation of medical imaging reports) as an academic solution to optimize medical imaging reports production. Such a project requires two layers: one engineering layer to build the automation process, and a second medical layer to determine domain-specific data models for each type of report. We describe here the medical layer of this project. Methods. We designed a reproducible methodology to identify -for a given medical imaging exam- mandatory fields, and describe a corresponding simple data model using validated formats. The mandatory fields had to meet legal requirements, domain-specific guidelines, and results of a bibliographic review on clinical studies. An UML representation, a JSON Schema, and a YAML instance dataset were defined. Based on this data model a form was created using Goupile, an open source eCRF script-based editor. In addition, a graphical display was designed and mapped with the data model, as well as a text template to automatically produce a free-text report. Finally, the YAML instance was encoded in a QR-Code to allow offline paper-based transmission of structured data. Results. We tested this methodology in a specific domain: computed tomography for urolithiasis. We successfully extracted 73 fields, and transformed them into a simple data model, with mapping to a simple graphical display, and textual report template. The offline QR-code transmission of a 2,615 characters YAML file was successful with simple smartphone QR-Code scanner. Conclusion. Although automated production of medical report requires domain-specific data model and mapping, these can be defined using a reproducible methodology. Hopefully this proof of concept will lead to a computer solution to optimize medical imaging reports, driven by academic research.
\end{abstract}

Keywords. Data model, medical imaging report, QR-Code, patient participation.

\footnotetext{
${ }^{1}$ Corresponding Author, Arthur LAURIOT DIT PREVOST, Clinique de Chirurgie et Orthopédie de l'Enfant, CHU Lille - Hôpital Jeanne de Flandre, Avenue Eugène Avinée, F-59000 Lille;E-mail: arthur.lauriotditprevost@chru-lille.fr.
} 


\section{Introduction}

Free text medical reports are the standard support for medical information. Medical imaging reports are the primary interface between the radiologist, the patient, and the prescribing physician. Improving radiological reporting practice is a growing area of interest in the medical literature, especially report's quality, format, language, length, content and completeness, turnaround time, and mode of result delivery to patients $[1,2]$. Medical information also plays a central role in physician-patient relationship, especially with the concept of patient empowerment [3]. Improving the patients' understanding of their conditions, results in a higher adherence to care [4]. This could be achieved using graphical display [5].

For that purpose, we initiated the ICIPEMIR project: "Improving the completeness, interoperability and patients' explanation of medical imaging reports". The objective is to design a workflow for producing domain-specific medical imaging reports based on upstream production of structured data by the radiologist and automated production of both textual report and schematic graphical representation. Expected benefits would be: improved completeness and clarity through synoptic reporting [6], higher patient understanding through schematic graphical representation of the result, faster report writing, and enhanced storage and sharing of structured data for future clinical research. Interoperability is also a crucial point when dealing with health data [7]. Although efforts were made towards interoperability standards in healthcare through initiatives like FHIR (Fast Healthcare Interoperability Resources) [8] or OpenEHR [9], these standards remains complex structures. Besides, as the traditional decentralized paper-based transmission has proven effective and safe for transmitting and storage of unstructured text-based data, we aim to explore the possibility of transmitting light weight structured data through this communication channel, with the use of QR-Codes.

Figure 1 presents the intended final use of ICIPEMIR products. The radiologist would fill-in a domain-specific form with pre-defined fields, to generate a printed imaging report composed of: (1) a standard text report, (2) a graphical display, and (3) a QR-Code embedding the structured data.

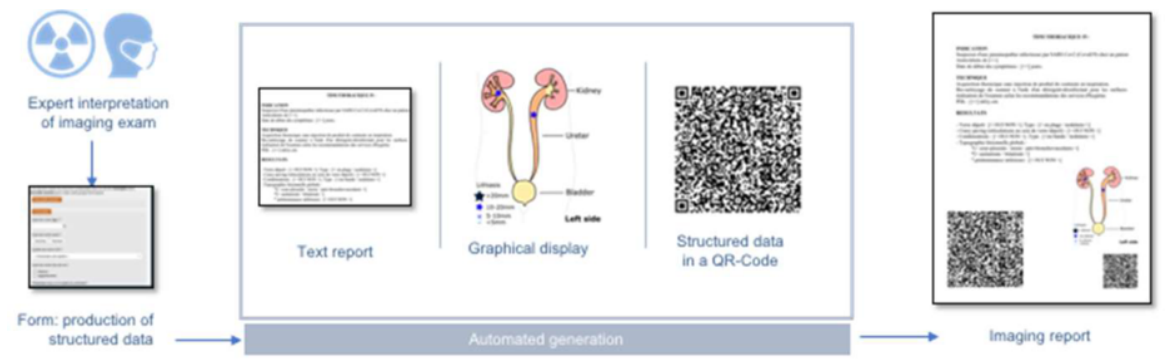

Figure 1. ICIPEMIR workflow for automated production of imaging report.

The QR-code would enable to safely store on the paper and transmit offline medical information. The QR-Code would embed any URL, but a small data file, to enable data sharing without security issue: as the patient is allowed to carry and transmit the paper report, he is also allowed to carry and transmit the encoded data. This first requires defining mandatory information, in the form of a specific data model, which can only be done for a given imaging exam, and a given indication. The objective of this paper is to describe our methodology to define the required information and structure them as a simple data model for computed tomography for urolithiasis. 


\section{Method}

\subsection{Field definition: literature-based definition of relevant fields with expert validation}

We conducted a narrative bibliographic review using the Medline database (on http://pubmed.gov), Google, and Google Scholar, to identify guidelines on medical imaging reporting in general, and more specifically related to targeted exam and indication (here computed tomography for urolithiasis). We also included legal requirement on medical reporting, by screening the French official health agency and health regulation codes. We also searched the Medline database for scientific papers reporting imaging results of patients suffering from urolithiasis. The objective was to identify the pieces of information the authors had found relevant to report.

The list of items to report was blindly reviewed by 4 independent experts from our university hospital (a radiologist, a general practitioner, a urologist, and a medical informatics specialist). A final consensus meeting was organized.

\subsection{Data model: standardization of the data collection within the report}

The list of fields was transformed into a simple data model (Figure 2) and modelized using UML [10]. We then defined a possible instance of the data model using the YAML syntax [11]. We also defined the corresponding JSON schema [12] for schema validation. Finally, we used the web-based open source e-CRF tool Goupile [13], to output a shareable easy-to-use form for any imaging report in the defined domain. The form was intended to output the data following the YAML syntax defined above. The YAML file was finally encoded into an automatically generated QR Code with a 7\% error. This format enables to embed up to 2,953 characters.

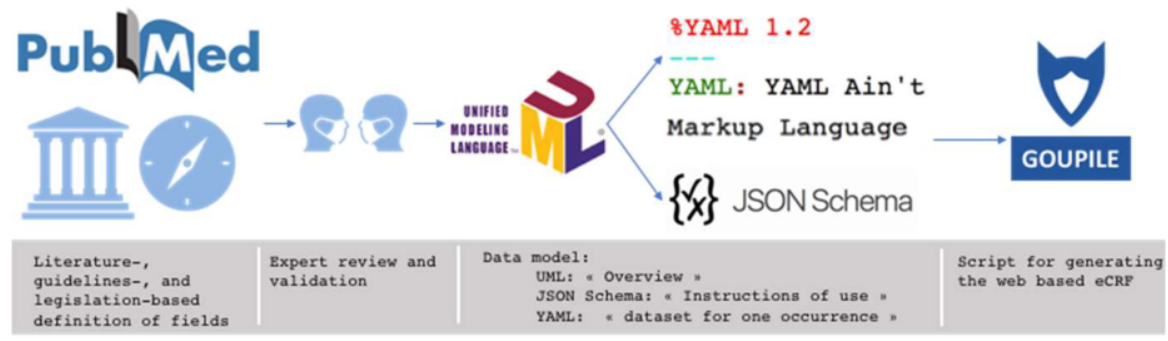

Figure 2. Iterative methodology for domain-specific data model definition.

\section{Results}

We tested this methodology on computed tomography for urolithiasis. The final model retained 73 fields (Figure 3), divided into 3 tables with different cardinality, that was handled by JSON and YAML syntax (e.g. the variable size of the lithiasis was likely to appear more than once in a single report, so we created a table for lithiasis related fields). A JavaScript file was also written to produce a form from Goupile. All result files can be found online on https://github.com/arthurldp/medical_imaging_report. The QR-Code containing a 2,615 character YAML file was successfully decoded by 4 out of 5 smartphone models, of which one model retrieved the data through the native camera application, and 3 through different non-native QR Code scanning apps. 


\begin{tabular}{|c|c|c|c|c|}
\hline \multirow{2}{*}{\multicolumn{2}{|c|}{ Exan }} & \multicolumn{3}{|c|}{ 0...* } \\
\hline & & \multicolumn{3}{|c|}{ LithiasisResults } \\
\hline \multirow{3}{*}{$\begin{array}{l}\text { nir } \\
\text { patient_first_name } \\
\text { patient_name } \\
\text { patient_birth }\end{array}$} & $\begin{array}{l}\text { : string } \\
\text { : string }\end{array}$ & lithiasis_id & \multicolumn{2}{|c|}{ : hash (PK) } \\
\hline & $\begin{array}{l}\text { : string } \\
\text { : date }\end{array}$ & \multirow{4}{*}{$\begin{array}{l}\text { evolution } \\
\text { lateralisation } \\
\text { location } \\
\text { coralliform }\end{array}$} & \multicolumn{2}{|c|}{ : categorical } \\
\hline & : date & & \multicolumn{2}{|c|}{ : categorical } \\
\hline $\begin{array}{l}\text { physician } \\
\text { institution }\end{array}$ & $\begin{array}{l}\text { : string } \\
\text { : string }\end{array}$ & & \multicolumn{2}{|c|}{ : categorical } \\
\hline exan_datetine & : date & & \multicolumn{2}{|c|}{ : categorical } \\
\hline exan_type & : categorical & size & \multicolumn{2}{|c|}{ : real } \\
\hline device_model & : string & density & \multicolumn{2}{|l|}{ : real } \\
\hline device_date & $\begin{array}{l}\text { : date } \\
\text { : categorical }\end{array}$ & uvj_distance & : real & \\
\hline \multirow{5}{*}{$\begin{array}{l}\text { condition_contrast } \\
\text { condition_lowdose } \\
\text { condition_acute } \\
\text { condition_fever } \\
\text { condition_derivation } \\
\text { condition_radiation }\end{array}$} & : categorical & infiltration & : categoric & ical \\
\hline & \multirow{2}{*}{$\begin{array}{l}\text { : categorical } \\
\text { : categorical }\end{array}$} & obstruction & : categori & ical \\
\hline & & nephrography & : categor & ical \\
\hline & $\begin{array}{l}\text { : categorical } \\
\text { : categorical }\end{array}$ & fornix_rupture & : categor & ical \\
\hline & : real & comnent & : string & \\
\hline abnornal_liver & : categorical & & & \\
\hline abnornal_adrenals & : categorical & & & \\
\hline abnormal_pancreas & : categorical & & & \\
\hline abnornal_spleen & : categorical & & & \\
\hline abnormal_ovaries & : categorical & & $0 . .8$ & \\
\hline abnormal_lymphnode & : categorical & Uri & ryTractResu & \\
\hline infiltration_gallbladder & $\begin{array}{l}\text { : categorical } \\
: \text { categorical }\end{array}$ & \begin{tabular}{|l} 
urinary_tract_ic \\
\end{tabular} & & : hash (PK) \\
\hline $\begin{array}{l}\text { infiltration_appendix } \\
\text { infiltration_sigmoid }\end{array}$ & $\begin{array}{l}\text { : categorical } \\
\text { : categorical }\end{array}$ & lateralisation & & : categorical \\
\hline pneunoperitoneum & : categorical & diversion & & : categorical \\
\hline intraperitoneal_effusion & : categorical & renal_height & & : real \\
\hline urinary_tract_duplication & : categorical & renal_volume & & : real \\
\hline renal_transplant & : categorical & renal_contour_de & ormity & : categorical \\
\hline pelvic_phlebolith & : categorical & renal_parenchyma & _anomaly & : categorical \\
\hline bladder_lithiasis & : categorical & interlabial_pelv & C_diameter & : real \\
\hline n_Lithiasis & : integer & extrasinusal_pe & & : categorical \\
\hline findings & : categorical & ureteral_dianete & & : real \\
\hline further_inaging & : categorical & ureteral_dilatat & & : categorical \\
\hline $\begin{array}{l}\text { further_imaging_type } \\
\text { further_physician }\end{array}$ & : string & pyelocaliceal_di & atation & : categorical \\
\hline $\begin{array}{l}\text { further_physician } \\
\text { further_physician_type }\end{array}$ & $\begin{array}{l}\text { : categorical } \\
: \text { string }\end{array}$ & pelviureteric_ju & ction & : categorical \\
\hline $\begin{array}{l}\text { further_physician_type } \\
\text { control_inaging }\end{array}$ & $\begin{array}{l}\text { : } 5 \text { tring } \\
: \text { categorical }\end{array}$ & urinoma & & : categorical \\
\hline control_imaging_delay & : string & hesaturia & & : categorical \\
\hline coment & : string & pyelonephritis_s & & : categorical \\
\hline conclusion & : string & \begin{tabular}{|l|} 
comment \\
\end{tabular} & & : string \\
\hline
\end{tabular}

Figure 3. UML schema of the "computed tomography urolithiasis report" data model

\section{Discussion and conclusion}

This paper intends to describe an optimal workflow for producing medical imaging report using structured data. The key point is to define mandatory fields to set a simple data model for each domain of application. We think this methodology is an efficient way of iteratively defining such data model, domain by domain, each within an academic research work. We already started to apply this methodology in other imaging exams.

We validated our fields list with only 4 experts. Ideally a larger panel of experts would be necessary. The main advantage of a "small size" expert panel is the high responsiveness, and a shorter time to consensus. For this reason, we decided to propose and publish a data model, and leave the possibility to adjust it later based on feedbacks from medical societies or editors' comments.

As for the rationale of our method in relation to pre-existing interoperability standards initiatives, we initially intended to define simple, light weight, and human readable data models. But it also appears relevant to use and integrate thereafter the successive "light weight" data models as archetypes in the OpenEHR platform $[9,14]$. Also whenever applicable, some selected elements from FHIR specification should be used in our data model (such as elements from the "ImagingStudy" resource and "MeasureReport" resource of HL7 FHIR v4)[8]. 
To the best of our knowledge, the use QR-Code to store and transfer structured medical data through the traditional paper-based communication channel has never been described. Recently, Mao et al. proposed a secure way of transmitting medical text data using a video stream of successive QR-Code [15]. We believe this concept can be adapted to the traditional paper-based transmission, and medical imaging report is an example of choice, because being highly domain-specific, it narrows size of the dataset file to be encoded. Also QR-Code scanning in clinical data warehouse might supposedly be more reliable than combining optic character recognition and natural language processing to detect structured data in clinical data warehouse. Further work on QR-Code storage of medical data will focus on compression and encryption methods, to lower the QR-Code size, and secure the access of these data.

We are planning to develop a software for producing medical report based on these tailored data models. This solution is intended to take as input the data model and the mappings, and to produce as an output an enhanced medical imaging report.

\section{References}

[1] Wynia MK, Osborn CY. Health literacy and communication quality in health care organizations. J Health Commun. 2010;15 Suppl 2(Suppl 2):102-15.

[2] McNair AG, Brookes ST, Davis CR, Argyropoulos M, Blazeby JM. Communicating the results of randomized clinical trials: do patients understand multidimensional patient-reported outcomes? J Clin Oncol. 2010 Feb 10;28(5):738-43.

[3] Manaouil C, Saliou G, Valléé JN, Jardé O. La loi du 4 mars 2002: comment l'appliquer en matière d'information des patients en radiologie [The law of March 4, 2002: how to implement it with regards to informed consent in radiology]. J Radiol. 2006 Apr;87(4 Pt 1):355-62.

[4] Mityul MI, Gilcrease-Garcia B, Searleman A, Demertzis JL, Gunn AJ. Interpretive Differences Between Patients and Radiologists Regarding the Diagnostic Confidence Associated With Commonly Used Phrases in the Radiology Report. AJR Am J Roentgenol. 2018 Jan;210(1):123-126.

[5] Bantug ET, Coles T, Smith KC, Snyder CF, Rouette J, Brundage MD; PRO Data Presentation Stakeholder Advisory Board. Graphical displays of patient-reported outcomes (PRO) for use in clinical practice: What makes a pro picture worth a thousand words? Patient Educ Couns. 2016 Apr;99(4):483490.

[6] Goel AK, DiLella D, Dotsikas G, Hilts M, Kwan D, Paxton L. Unlocking Radiology Reporting Data: an Implementation of Synoptic Radiology Reporting in Low-Dose CT Cancer Screening. J Digit Imaging. 2019 Dec;32(6):1044-1051.

[7] Lehne M, Sass J, Essenwanger A, Schepers J, Thun S. Why digital medicine depends on interoperability. NPJ Digit Med. 2019 Aug 20;2:79.

[8] HL7 FHIR®. Fast Healthcare Interoperability Resources. Available at https://www.h17.org/implement/standards/product_brief.cfm?product_id=491. Accessed 2019.

[9] OpenEHR International. Open industry specifications, models and software for e-health. Available at https://www.openehr.org/. Accessed 2020.

[10] OMG. Object Management Group. Unified Modelling Language (UML). Available at https://www.omg.org/spec/UML/About-UML. Accessed 2017.

[11] Ben Kiki O, Evans C, döt Net I. YAML Ain’t Markup Language (YAML). 2009. Available at https://yaml.org/spec/1.2/spec.html. Accessed 2020.

[12] Wright A, Andrews H, Hutton B. JSON Schema. 2019. Available at https://json-schema.org/draft/201909/json-schema-core.html. Accessed 2020.

[13] Martignene N, Lamer A. Goupile. Goupile 2020. Available at: https://goupile.fr. Accessed October 6, 2020.

[14] Sousa M, Ferreira D, Santos-Pereira C, Bacelar G, Frade S, Pestana O, et al. openEHR Based Systems and the General Data Protection Regulation (GDPR). Stud Health Technol Inform 2018;247:91-5.

[15] Mao H, Chi C, Yu J, Yang P, Qian C, Zhao D. QRStream: A Secure and Convenient Method for Text Healthcare Data Transferring. 2019 41st Annu. Int. Conf. IEEE Eng. Med. Biol. Soc. EMBC, Berlin, Germany: IEEE; 2019, p. 3458-62. 